\title{
The Horizon for Treating Cutaneous Vascular Lesions
}

\author{
Amit M. Patel, MD, ${ }^{*}$ Elizabeth L. Chou, BS, ${ }^{*}$ Laura Findeiss, MD, ${ }^{\dagger}$ and Kristen M. Kelly, MD*
}

\begin{abstract}
Dermatologists encounter a wide range of cutaneous vascular lesions, including infantile hemangiomas, port-wine stain birthmarks, arteriovenous malformations, venous malformations, Kaposi sarcomas, angiosarcomas, and angiofibromas. Current treatment modalities to reduce these lesions include topical and/or intralesional steroids, laser therapy, surgical resection, and endovascular therapy. However, each method has limitations owing to recurrence, comorbidities, toxicity, or lesion location. Photodynamic therapy, antiangiogenic therapy, and evolving methods of sclerotherapy are promising areas of development that may mitigate limitations of current treatments and offer exciting options for patients and their physicians.

Semin Cutan Med Surg 31:98-104 @ 2012 Elsevier Inc. All rights reserved.
\end{abstract}

KEYWORDS vascular malformations, infantile hemangiomas, port-wine stain, Kaposi sarcoma, angiosarcomas, angiofibromas, photodynamic therapy, angiogenesis inhibitors, sclerotherapy

$\mathrm{D}$ ermatologists encounter a wide range of cutaneous vascular lesions, including infantile hemangiomas (IHs), portwine stain birthmarks (PWS), arteriovenous malformations (AVMs), venous malformations (VMs), Kaposi sarcomas (KS), angiosarcomas, and angiofibromas (Table 1). This article provides a brief introduction to these entities, including current treatment options and limitations of these therapies. We then look forward and describe developing treatment modalities, including photodynamic therapy (PDT), antiangiogenic therapy, and evolving methods of sclerotherapy (ST), which offer exciting potential for more complete and effective removal of cutaneous vascular lesions.

\section{Infantile Hemangiomas}

IHs are the most common benign vascular tumors in children and are present at birth or become evident during the first sev-

\footnotetext{
*Department of Dermatology, University of California, Irvine, CA. † Department of Interventional Radiology, University of California, Irvine, CA. Conflict of Interest Disclosures: The authors have completed and submitted the ICMJE Form for Disclosure of Potential Conflicts of Interest. Dr. Kelly's institution has received a grant from Graceway Pharmaceuticals for work mentioned in this manuscript. Dr. Kelly's institution has received grants or has grants pending with Graceway Pharmaceuticals, New Star Lasers/Cool Touch, and Candela Corporation. Dr. Findeiss has received payment for service on speakers bureaus from Bayer Pharmaceuticals for use of sorafenib in hepatocellular carcinoma. Dr. Patel and Ms. Chou have no conflicts to report.

Address reprint requests to Kristen M. Kelly, MD, Department of Dermatology, University of California, 1002 Health Sciences Rd, Irvine, CA 92612.E-mail: KMKelly@uci.edu
}

eral weeks of life. They may be superficial (capillary: typically presenting as bright-red papules, plaques), deep (subcutaneous nodules), or have both components. Natural history of $\mathrm{IH}$ is rapid proliferation over the course of months, followed by involution over the course of years. As such, many hemangiomas are managed conservatively, with close observation for the development of complications. Treatment is considered for lesions that impair vital functions, are ulcerated, or present on areas likely to leave significant deformity after involution.

Treatment options include topical treatments (most commonly topical steroids), intralesional steroids, laser, and surgical excision. Until recently, the most commonly implemented oral treatment for hemangiomas was corticosteroids. In the past few years, oral propranolol, a beta-blocker, has been used with increasing frequency and has revolutionized treatment for these lesions. Effects of treatment vary by patient and lesion, including a slow or incomplete response in some cases. Potential side effects associated with systemic medications include irritability, gastric upset, transient growth retardation, adrenal suppression, and immunosuppression for corticosteroids and hypotension, bradycardia, hypoglycemia, bronchospasm, and hyperkalemia for propranolol.

\section{Port-Wine Stain Birthmarks}

PWSs are vascular malformations found in approximately $0.3 \%$ of newborns and are composed of ectatic capillaries and postcapillary venules in the superficial vascular plexus. Vessels vary in size from $10 \mu \mathrm{m}$ to $150 \mu \mathrm{m}$. Although they may occur anywhere, they are commonly found on the head and neck. PWSs persist throughout life 
Table 1 Current and Promising Investigational Treatment Modalities for Cutaneous Vascular Lesions

\begin{tabular}{|c|c|c|c|c|}
\hline \multirow[b]{2}{*}{ Lesion } & \multirow{2}{*}{$\begin{array}{l}\text { Current Common Treatment } \\
\text { Modalities }\end{array}$} & \multicolumn{3}{|c|}{ Promising Modalities Under Investigation } \\
\hline & & PDT & Antiangiogenic Therapy & Novel Sclerotherapy \\
\hline $\mathrm{IH}$ & $\begin{array}{l}\text { Topical steroids, intralesional } \\
\text { steroids, laser therapy, } \\
\text { surgical excision, oral } \\
\text { steroids, propranolol }\end{array}$ & & $\begin{array}{l}\text { Imiquimod, propranolol, } \\
\text { topical beta-blocker }\end{array}$ & \\
\hline PWS & PDL & $\begin{array}{l}\text { PDT alone or } \\
\text { combined } \\
\text { with PDL }\end{array}$ & $\begin{array}{l}\text { PDL followed by imiquimod, } \\
\text { rapamycin, or other } \\
\text { angiogenesis inhibitors }\end{array}$ & \\
\hline AVM & $\begin{array}{l}\text { Surgical resection, embolization, } \\
\text { sclerotherapy, or combination }\end{array}$ & $\begin{array}{l}\text { Interstitial } \\
\text { PDT }\end{array}$ & Marimastat & \\
\hline VM & $\begin{array}{l}\text { Surgical resection, } \\
\text { sclerotherapy, laser therapy }\end{array}$ & $\begin{array}{l}\text { Interstitial } \\
\text { PDT }\end{array}$ & & $\begin{array}{l}\text { Novel sclerosants, post- } \\
\text { treatment saline } \\
\text { dilution }\end{array}$ \\
\hline KS (non-HIV) & $\begin{array}{l}\text { External beam radiation, surgical } \\
\text { excision, cryotherapy, laser } \\
\text { ablation, intralesional } \\
\text { chemotherapy, topical } \\
\text { retinoids }\end{array}$ & PDT & $\begin{array}{l}\text { Rapamycin, imiquimod, } \\
\text { thalidomide, lenalidomide, } \\
\text { bevacizumab, sorafenib }\end{array}$ & \\
\hline Angiofibroma & $\begin{array}{l}\text { Ablative } \mathrm{CO}_{2} \text {, or erbium:yttrium- } \\
\text { aluminum-garnet laser }\end{array}$ & & $\begin{array}{l}\text { Rapamycin (oral and } \\
\text { topical) }\end{array}$ & \\
\hline Angiosarcoma & $\begin{array}{l}\text { Surgical excision, } \\
\text { chemotherapy, or combination }\end{array}$ & PDT & $\begin{array}{l}\text { Bevacizumab alone or in } \\
\text { combination with } \\
\text { destructive therapy }\end{array}$ & \\
\hline
\end{tabular}

IH, infantile hemangioma; PWS, port-wine stain birthmark; PDL, pulsed dye laser; PDT, photodynamic therapy; AVM, arteriovenous malformation; VM, venous malformation; KS, Kaposi sarcoma; $\mathrm{CO}_{2}$, carbon dioxide; VEGF, vascular endothelial growth factor.

and, over decades, may develop soft-tissue overgrowth and nodules, which may bleed. These lesions are often considered disfiguring, and many patients or their families seek treatment.

In the United States, lasers are the gold standard for PWS removal. The pulsed dye laser (PDL) is used most commonly, but other light-based devices, including Alexandrite lasers, intense pulsed light, and combined 595/1064-nm devices, are used to achieve desired lesion lightening. Usually more than 10 treatment sessions are required and complete lesion lightening may not occur. Multiple factors limit laser treatment efficacy. First, competitive absorption of therapeutic laser light by epidermal melanin reduces the light dose reaching target blood vessels. This limitation is particularly significant among patients with darker skin types. Second, small superficial vessels ( $<20 \mu \mathrm{m}$ in diameter) rapidly diffuse the heat induced by laser therapy and are thus difficult to photocoagulate. Third, revascularization may occur owing to neovascularization and reperfusion of partially damaged vessels.

\section{Arteriovenous Malformations}

AVMs are fast-flow vascular lesions created by the direct joining of arterial and venous systems without an intermediate capillary bed. Although the etiology is unclear, they are believed to result from abnormal vascular development during embryogenesis. AVMs may involve any organ system. Cutaneous AVMs may cause disfigurement, destruction of tissue, pain, bleeding, or ulceration. ${ }^{1}$
Treatment involves complete destruction of the nidus or fistula of the AVM through surgical resection, endovascular therapy, or a combination of both techniques. Combined therapy, characterized by preoperative embolization, enables a more complete resection and reduces intraoperative blood loss and postoperative morbidity and mortality. Embolization alone may be definitive for patients with high surgical risks or surgically inaccessible AVMs. This type of therapy requires multiple sessions and precise delivery of treatment agents into the nidus. Limitations of current treatments include recurrence due to incomplete resection, recanalization, or parasitization of new vessels; cardiopulmonary complications; and disfigurement.

\section{Venous Malformations}

VMs are nonproliferating low-flow malformations composed of anomalous ectatic vascular channels. These soft, often "bluish," compressible masses are the most common of all symptomatic vascular lesions and are present at birth. Although most arise sporadically and are unifocal, approximately $1 \%$ of lesions are multifocal (including a majority of the inherited forms of VMs). Lesions can grow over time, primarily during the childhood years, and can be associated with pain. ${ }^{2}$

Current treatments include surgical resection, fluoroscopic-guided ST, or a combination of the 2 , as well as percutaneous and intralesional laser therapy. Surgical resection is often limited by the size and location of the lesions. There is a significant risk of bleeding and spontaneous thrombosis/ 
thrombolysis or disseminated intravascular coagulation with larger VMs. ST reduces lesion size by introducing chemical irritants into the lumen of a vascular structure, with the intent of promoting endothelial damage and obliteration of the vessel. Absolute ethanol is a commonly used sclerosant, owing to its overall effectiveness in obliterating target vessels and low rates of lesion recurrence. ${ }^{3}$ Side effects include nontarget delivery of agent causing local nerve damage along with skin ulceration, blistering, and necrosis, in addition to systemic complications such as hemoglobinuria, hemolysis, renal failure, myocarditis, acute pulmonary hypertensive crisis, and cardiovascular collapse. Although laser therapy of VMs is associated with fewer complications compared with surgical resection and ST, it may be less efficacious, especially for larger lesions, and recurrence is not uncommon. Heat generated by laser therapy may also cause thermal damage to the epidermis and dermis.

\section{Kaposi Sarcoma}

$\mathrm{KS}$ is an angiogenic tumor thought to be caused by infection or reactivation of human herpes virus 8 , also known as Kaposi sarcoma herpes virus. Clinically, the cutaneous lesions are violaceous-to-red-colored macules and papules that typically coalesce to form patches and plaques. There are several types of KS, including epidemic acquired immune deficiency syndrome (AIDS)-related KS, immunocompromised KS, classic KS, and the endemic African KS subtype. All, however, are angioproliferative disorders characterized by endothelial cell proliferation, infiltration of inflammatory cells, activation of angiogenic cellular pathways, and vascular endothelial growth factor (VEGF) dysfunction.

In human immunodeficiency virus (HIV)-positive patients, both protease inhibitors and non-nucleoside transcription inhibitors seem to have a protective role secondary to the inhibition of viral replication. For HIV-associated KS, lesions may resolve with multidrug HIV therapy. Current treatment options for non-HIV-associated or resistant lesions include external beam radiation, surgical excision, cryotherapy, laser ablation, intralesional chemotherapy, and topical retinoids. Recurrence is common, especially for medical regimens that often confer resistance.

\section{Angiosarcomas}

Angiosarcomas are rare malignant vascular tumors arising from endothelial cells in the blood or lymphatic vessels. They present as bruiselike areas that ulcerate or become nodular, with small satellite lesions next to the main tumors. These tumors do not have a capsule or a clear border separating normal and abnormal tissue. They may occur anywhere in the body, but commonly involve the head and neck region, particularly the scalp. Although most arise spontaneously, previous radiotherapy treatment is an independent risk factor. ${ }^{4}$ The infiltrative and aggressive nature of angiosarcomas confer a poor prognosis, with an overall 5-year survival of approximately $35 \% .^{5}$

Size of primary lesion and presence or absence of distant metastases help determine treatment options. In the absence of metastasis, radical surgery with complete resection is the most common treatment, but incomplete resection and recurrence is frequent. Cytotoxic chemotherapy is the primary treatment option for metastatic angiosarcomas, but owing to the advanced age of most patients, the treatment is limited by risk of toxicity and comorbidities.

\section{Angiofibromas}

Angiofibromas are cutaneous manifestations of tuberous sclerosis (TS). TS is an autosomal dominant disorder resulting from defects in tuberous sclerosis complex (tsc) genes 1 and 2. tsc 1 and 2 produce hamartin and tuberin complex proteins that inhibit the mammalian target of rapamycin (RPM) pathway. tsc mutations cause dysfunction of inhibitory proteins and result in abnormal growths and blood vessel proliferation in several organs. Facial angiofibromas are seen in $70 \%$ to $80 \%$ of patients and may be disfiguring. They do not resolve without treatment. Treatment options include ablative carbon dioxide or erbium:yttrium-aluminum-garnet laser therapy. Dramatic improvement can be achieved, but lesions often recur within months.

\section{New and Developing Treatment Modalities}

\section{Photodynamic Therapy}

PDT involves light activation of a photosensitizer, which reacts with local oxygen to generate cytotoxic reactive oxygen species and subsequent tissue injury which may include blood vessel wall damage or destruction. PDT is currently used to treat a wide range of benign, premalignant, and malignant conditions and has promising potential for treatment of PWS, AVMs, VMs, KSs, and angiosarcomas.

PDT has advantages and disadvantages when compared with other modalities used worldwide. The low optical powers (mW) associated with PDT do not cause epidermal injury, allowing treatment of all skin types. Further, PDT uses continuous low irradiance light over long exposures (several minutes), enabling a cumulative effect at progressively deeper regions as exposure time is increased. Thus, treatment can cause vascular injury in vessels of all sizes and lesions of various depths. Selection of intravenously administered photosensitizers, as opposed to topical or oral, permits selective removal of blood vessels in carefully designed protocols. PDT, however, has not been openly embraced by most of the world owing to the prolonged photosensitivity (1-4 weeks) and significant risk of scarring associated with current treatment protocols. Innovative PDT protocols may address these limitations and enhance benefits. In addition, new photosensitizers with shorter photosensitivity periods are currently available and using these agents may improve treatment outcomes.

To date, clinicians in China have the most extensive experience using PDT for treatment of cutaneous vascular lesions. In 2007, Qin et al. ${ }^{6}$ presented data on 238 patients with PWS 
who underwent photocarcinorin (PsD-007; Second Military Medical Univeristy, Shanghai) PDT using a copper vapor laser (Model IECu-10, Beijing Kedian Microwave Electronics Co. Ltd.) (peak spectral output at $510.6 \mathrm{~nm}$ and $578.2 \mathrm{~nm}$ ). After 2 to 4 PDT treatment sessions, they reported excellent results in 29\% of the cases, good results in 32\%, fair response in 37\%, and poor response in 3\%. More recently, Qiu et al. ${ }^{7}$ reported findings from their long-term study of 1385 PWS patients treated with 3 different photosensitizers (hematoporphyrin monomethyl ether, hematoporphyrin derivative sodium, or PsD-007 [3-7 mg/kg]), followed by argon laser irradiation (power density: 50-100 $\mathrm{mW} / \mathrm{cm}^{2}$ ). After only 1 PDT treatment session, $6.6 \%$ of patients achieved excellent results, $38.3 \%$ good results, $47.4 \%$ fair results, $7.4 \%$ poor results, and $0.5 \%$ reported no visible change. ${ }^{7}$ Patients in both studies were required to avoid sun exposure for up to 4 weeks.

Our group has combined PDT and PDL in an effort to overcome the limitations of either therapy. PDT followed by PDL combines the photochemical and photothermal aspect of each therapy to enhance the vascular damage caused by PDT. The combination also lowers total radiant exposure during therapy and minimizes adverse effects such as scarring. ${ }^{8}$ Preliminary basic science research demonstrated the benefits of this approach using benzoporphyrin derivative monoacid ring A as the photosensitizer and 576-nm continuous-wave light. ${ }^{9}$ A clinical dose-response study was then performed with the same protocol among 8 patients in 11 treatment sites. ${ }^{8}$ Treatments were well tolerated; subjects did not report any increased discomfort during combined PDT + PDL as compared with PDL treatment. Adverse effects were limited to fine scabbing and temporary mild hyperpigmentation at PDL sites, which resolved without treatment. Subjects were required to avoid sun exposure for 5 days. Starting at a PDT radiant exposure of $75 \mathrm{~J} / \mathrm{cm}^{2}$, improved efficacy was noted in the combined PDT and PDL site, as near complete blanching was achieved. Additional studies are underway to improve this protocol using additional photosensitizers and light sources.

PDT has also been used for treatment of AVMs and VMs, especially for cases where resection is not possible owing to large lesions or proximity to vital anatomical structures. Interstitial PDT may improve treatment outcomes of deep malformations that sit beyond the $1-\mathrm{cm}$ penetrance of traditional PDT delivered by surface illumination. In 2011, Jerjes et al. ${ }^{10}$ reported outcomes of interstitial PDT for the treatment of 43 patients with vascular anomalies, 11 of whom had AVMs and 8 of whom had VMs. Under general anesthesia, patients were administered $0.15 \mathrm{mg} / \mathrm{kg}$ of meta-tetra-hydroxyphenyl chlorine 96 hours before treatment with an interstitial 652-nm diode laser (fluence of $10-20 \mathrm{~J} / \mathrm{cm}^{2}$ and fluence rate of 100 $\mathrm{mW} / \mathrm{cm}^{2}$ ). Intraoperatively, insertion of 1 or more needles into the lesion was guided by ultrasonography and followed by insertion of thin optical fibers to fully illuminate deep target tissues and activate the photosensitizer. Radiological assessment of post-treatment responses included 15 patients exhibiting a $50 \%$ to $75 \%$ reduction in malformation size, 11 with $<50 \%$ reduction, 12 with $<25 \%$ reduction, and 5 pa- tients exhibiting little to no change in lesion size. Jerjes et al. ${ }^{10}$ noted that lymphatic malformations and AVMs responded most favorably. PDT may help reduce lesion size before resection and reduce risk of intraoperative complications. Compared with standard resection and endovascular therapy, PDT for AVMs and VMs is minimally invasive, maintains the integrity of surrounding tissues and nerves, and can be repeated without risk of cumulating toxicity.

PDT may also be a promising treatment modality for KS and angiosarcoma. PDT-induced vascular damage targets tumor tissue and activates cell-mediated immune responses against tumor cells to enhance tumor eradication. In 2006, Tardivo et al. ${ }^{11}$ evaluated PDT (RL50, 600-750 nm) for treatment of AIDS-related KS in a patient with multiple lesions who did not achieve favorable outcomes after multiple rounds of chemotherapy. The patient received local and superficial injection of $2 \%$ methylene blue, $2 \%$ toluidine blue, and $2 \%$ lidocaine, followed by light irradiation with a noncoherent light source RL50 (600-750 nm; home-made, polychromatic spectral output of 600-750 nm, spot diameter of $45 \mathrm{~mm}, 100 \mathrm{~mW} / \mathrm{cm} 2$ ) at a total dose of $18 \mathrm{~J} / \mathrm{cm}^{2}$. After 4 months of weekly PDT, followed by 6 months of treatment every 2 weeks at the same site, the patient experienced complete remission with excellent cosmetic result. In 2007, SooPing Thong et al. ${ }^{12}$ reported the successful treatment of recurrent angiosarcoma in a 64-year-old Chinese patient using Fotolon (Haemato-science GmbH, Luckenwalde, Germany) (1:1 ratio of chlorin e6-polyvinylpyrrolidone [Ce6-PVP] and polyvinylpyrrolidone)-mediated PDT. Photosensitizer was administered intravenously at $2.0-5.7 \mathrm{mg} / \mathrm{kg}$ body weight for 10-20 minutes, followed by irradiation 3 hours later at 665 $\mathrm{nm}$ for a total light dose of $65-200 \mathrm{~J} / \mathrm{cm}^{2}$ at a rate of $80-150$ $\mathrm{mW} / \mathrm{cm}^{2}$. The patient achieved remission after 8 treatment sessions over 21 months. A new formulation of Ce6-PVP photosensitizer was evaluated in 2008 by Chin et al. ${ }^{13}$ in 3 patients with angiosarcoma. Fluorescence imaging allowed clear demarcation of cancerous tissue and demonstrated the preferential accumulation of Ce6-PVP in tumor cells. These promising outcomes in the treatment of KS and angiosarcomas with PDT may be enhanced by future investigation of photosensitizers and light dosages to optimize treatment protocols.

\section{Antiangiogenic Agents}

Antiangiogenic agents collectively refer to a family of inhibitory drugs that disrupt processes that would otherwise promote blood vessel growth and regeneration. In addition to its utility in cancer therapy, angiogenesis inhibition has become increasingly important in the treatment of cutaneous vascular lesions such as IHs, PWSs, AVMs, KS, angiosarcomas, and angiofibromas.

Although use of antiangiogenic agents for $\mathrm{IH}$ was first reported by Martinez et al. ${ }^{14}$ in 2002 with application of imiquimod, results were inconclusive, and in later years, betablockers became the focus of investigation. The mechanism of beta-blocker treatment in IH is unknown and under intense study. Antiangiogenic, anti-beta-adrenergic, and pro- 
apoptotic effects have been proposed. Using an in vitro explant model, Itinteang et al..$^{15}$ recently demonstrated that a population of $\mathrm{CD}_{3} 4^{+}$endothelial progenitor cells of the microvasculature found in proliferating IH expresses angiotensin-converting enzyme and angiotensin II receptor 2 , but not angiotensin II receptor 1 . It is thought that mesenchymal cells found in IH release VEGF in response to angiotensin II stimulation. Given that beta-blockers have long been known to suppress release of renin from the kidney, these findings offer yet another mechanism, as shown in Figure 1, by which beta-blockers exert their beneficial effects. Furthermore, the implication of the renin-angiotensin pathway in the pathogenesis of IH warrants a closer look at the potential roles of angiotensin-converting enzyme inhibitors, angiotensin II receptor 2 antagonists, and novel vasopeptidase inhibitors, such as omapatrilat, for treatment of $\mathrm{IH}^{16}$

Topical beta-blockers may reduce the side effects associated with systemic beta-blockers in the treatment of IH. Ni et al. ${ }^{17}$ first reported the efficacy of topical timolol in treating a superficial capillary hemangioma in a 4-month-old infant. They have since successfully treated 7 patients using a topical regimen consisting of $0.5 \%$ timolol maleate solution twice a day (for 1 to 6 months) and report a fading of color and decrease of tumor size and volume ranging from 55\% to 95\% in all 7 patients. No adverse effects were noted. These findings have prompted ongoing randomized controlled studies investigating the efficacy of topical beta-blockers in treatment of superficial $\mathrm{IH}$.

Laser destruction of blood vessels followed by antiangiogenic treatment to prevent vessel recurrence is a promising approach for treatment of PWS. Tremaine et al. ${ }^{18}$ used laserinduced selective photothermolysis with post-treatment application of imiquimod and found a slightly, but statistically significant, enhanced lightening with combination therapy compared with PDL alone. Phung et al. ${ }^{19}$ demonstrated that topical application of RPM after PDL significantly reduced perilesional revascularization of the PWS compared with PDL alone. They proposed that RPM downregulates hypoxiainducible factor- $1 \alpha$, a transcription factor that regulates VEGF expression. They also reported a deeper level of vascular necrosis after PDL plus RPM for a given light dose. Additional studies investigating the mechanism of vessel maintenance in PWS and combined laser/anti-angiogenic therapy will likely lead to improved treatment effects.

AVM treatment outcomes may be improved by antiangiogenic therapy as well. Proangiogenic hormones (estrogen,

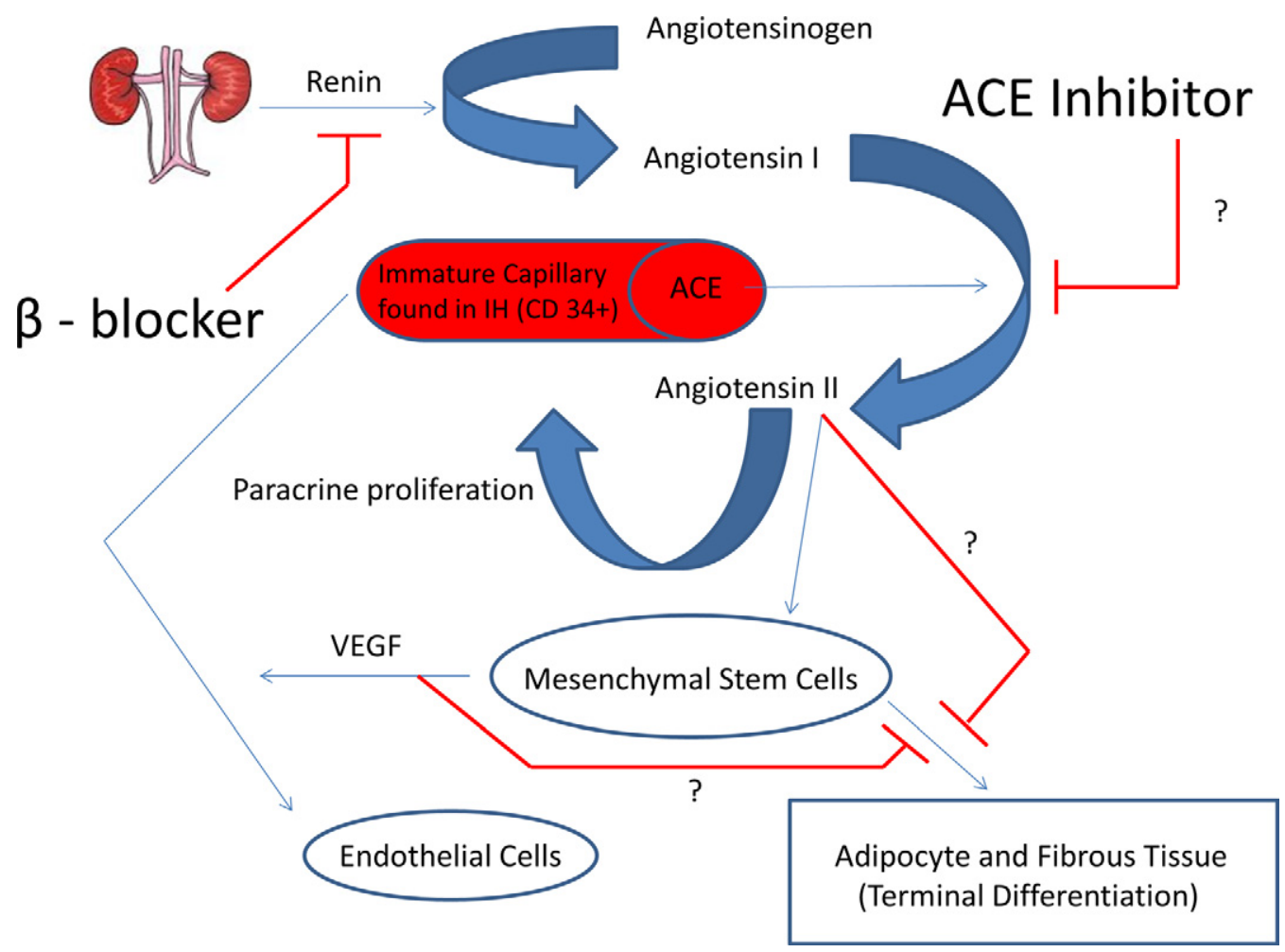

Figure 1 Proposed mechanism of action of beta-blockers in the treatment of IH. A CD34+ population of ICC found in $\mathrm{IH}$ are thought to express ACE in addition to the angiotensin II receptor. Local angiotensin I (AI) is converted by ACE to angiotensin II (A2). A2 presumably promotes: (a) the release of VEGF from MSC within IH, and (b) a paracrinelike proliferation of ICC. It is thought that VEGF not only prevents the terminal differentiation of MSC into fibrofatty tissue, but also facilitates the maturation of ICC into endothelial cells. Beta-blockers inhibit the upstream release of renin, thereby blocking the entire sequence of events illustrated previously. Note that in this proposed schematic, inhibition of VEGF release would lead to normal terminal differentiation of MSC into fibrofatty tissue, the clinically observed endpoint of IH. ACE, angiotensin-converting enzyme; IH, infantile hemangioma; ICC, immature capillary cells; VEGF, vascular endothelial growth factor; MSC, mesenchymal stem cells. 
progesterone, testosterone) released during puberty may account for the high risk of AVM expansion during that period. ${ }^{1}$ Burrows et al. ${ }^{20}$ recently showed that inhibiting angiogenesis through oral administration of marimastat, a matrix metalloproteinase inhibitor, effectively controlled a diffuse AVM that was unresponsive to endovascular treatment. During 12 years of treatment, the patient observed pain reduction, healing of lytic lesions, and improved limb function previously hindered by the AVM. This clinical observation supports speculation that inhibition of angiogenesis may alleviate AVM-related morbidity, expansion, and recurrence in humans ${ }^{21}$ and warrants further study.

Several agents with known antiangiogenic properties, including RPM, imiquimod, and thalidomide, have been used for treatment of KS. ${ }^{22}$ The efficacy, safety profile, and optimal oral dose of lenalidomide, an immunomodulatory and antiangiogenic agent, are under current investigation for treatment of HIV-associated KS treatment in a phase I/II study. It is believed to have fewer neuropathic side effects compared with thalidomide, a related drug shown to be somewhat efficacious in the treatment of chemotherapy-resistant AIDSrelated and classic KS. ${ }^{23-25}$ The authors of this multicenter trial are currently recruiting participants and anticipate completion by September 2013

Trials investigating VEGF inhibitors such as sorafenib and bevacizumab are currently underway. While sorafenib is being evaluated as a stand-alone therapy, bevacizumab is being studied in combination with pegylated liposomal doxorubicin. ${ }^{25}$ Bevacizumab has been used for treatment of angiosarcoma when surgical treatment is not possible owing to extent of disease and/or patient comorbidity. Koontz et al. ${ }^{26}$ reported findings from 2 patients with angiosarcoma of the nose who were treated with combination bevacizumab and radiotherapy before surgical resection. Pathologic evaluation showed no residual angiosarcoma and no recurrence at follow-up (8.5 months for one and 26 months for the other). Another case report described partial response (PR) after bevacizumab treatment of an extensive facial angiosarcoma, but rapid relapse occurred. ${ }^{27}$ Recently, De Yao et al. ${ }^{28}$ reported a biopsy-confirmed remission in an elderly patient with unresectable angiosarcoma of the scalp after combination radiotherapy and intravenous bevacizumab. Thus, combination destructive and antiangiogenic therapy may be a more promising approach for treatment of angiosarcomas.

In 2008, Hofbauer et al. ${ }^{29}$ described the improvement of facial angiofibromas in a patient with TS receiving oral RPM as part of her renal transplant immunosuppressive regimen. Owing to systemic side effects of oral RPM, clinicians investigated topical RPM. Haemel et al. ${ }^{30}$ used a 1\% RPM ointment applied twice daily for 3 months and reported striking improvement in reduction of lesion number and facial erythema after 6 weeks of follow-up. Systemic absorption of RPM was minimal, and no adverse side effects or aberrant lab findings were noted during the course of treatment. Another recent case series evaluated $0.4 \%$ RPM ointment administered 3 times a week for 9 months in 10 patients. The authors reported a reduction of the mean facial angiofibroma severity index (a scale developed to quantify and assess clinical im- provement) by $60.2 \%$. Median time to response was 4 weeks, and at the end of treatment, lesion size and extension in addition to erythema were reduced in all patients. ${ }^{31}$ Continuous application of medicine may be required, as recurrence has been reported after treatment cessation.

\section{Sclerotherapy}

The development of safer and more effective sclerosing compounds and delivery methods may improve treatment outcome for VMs. Newer detergent-based compounds such as sodium tetradecyl sulfate and polidocanol have satisfactory efficacy and improved safety profiles compared with ethanol.

Recently, Dompmartin et al. investigated the safety and efficacy of ethylcellulose-based gelified ethanol as a sclerotherapeutic agent in the treatment of VMs in a prospective study of 44 patients with mucosal, cutaneous, subcutaneous, and/or muscular slow-flow vascular malformations. Currently, this agent is used in Europe but is not available in the United States. Aesthetic improvement was noted by $81 \%$ of patients, whereas investigators noted at least some form of improvement in $91 \%$ of the patients. Local side effects, including necrosis with and without discharge of sclerosant, were reported in only $16 \%$ of patients and only in lesional areas with highest risk for localized complications (ie, face, lips, oral cavity, hands, and feet). Systemic side effects were not observed in any of the 44 patients. The authors attributed decreased number of adverse effects to the lower total alcohol content, increased viscosity, and decreased tissue permeability of the gelified ethanol. Furthermore, they found the gel to be at least as effective on a per-volume basis as absolute ethanol in the treatment of slow-flow vascular malformations and speculated that higher doses of the gelified compound can be safely used to treat even high-risk lesions. ${ }^{32}$

Ihara et al. ${ }^{33}$ recently looked at the effects of post-ST injection of saline in the subcutaneous space on localized skin necrosis and inflammation. Using an in vitro cytotoxicity assay, they first demonstrated that a 1:10 dilution with saline reduced the cytotoxicity of ethanol against fibroblasts and keratinocytes. Then, using a rabbit vein sclerosant retention model, they showed that subcutaneous injection of saline after ST with ethanol on an equal-volume basis prevented the otherwise expected gross cutaneous alterations and histologic presence of perivascular inflammatory infiltrates associated with ethanol ST. Interestingly, obstructive venous thrombus formation was not affected by post-treatment saline injection over 7 days of observation. The authors proposed that the observed protective effects may be due to the dilution of ethanol leaking into surrounding tissues, mechanical separation of the vein and skin, increased flow of interstitial fluid, and increased removal of ethanol from the dermal-epidermal space. Future studies investigating the clinical applicability of this technique for treatment of VMs are warranted.

\section{Conclusions}

Dermatologists encounter a wide range of cutaneous vascular lesions that may be disfiguring and/or life threatening. Cur- 
rent treatment modalities have varying success in obliterating these lesions owing to treatment toxicity, lesion location, and recurrence. PDT, antiangiogenic therapy, and novel ST methods and agents may reduce the limitations of current treatments. Future investigation of these options and ultimately safe incorporation into practice may offer our patients greatly improved treatment outcomes.

\section{Acknowledgment}

The work was supported in part by grants obtained from the National Institutes of Health (AR51443; HD065536), the National Institutes of Health Laser Microbeam and Medical Program (LAMMP, a P41 Biotechnology Resource, project number RR001192), and the Arnold and Mabel Beckman Foundation

\section{References}

1. Liu AS, Mulliken JB, Zurakowski D, et al: Extracranial arteriovenous malformations: Natural progression and recurrence after treatment. Plast Reconstr Surg 125:1185-1194, 2010

2. Garzon MC, Huang JT, Enjolras O, et al: Vascular malformations: Part I. J Am Acad Dermatol 56:353-370, 2007; quiz 71-74

3. Rimon U, Garniek A, Galili Y, et al: Ethanol sclerotherapy of peripheral venous malformations. Eur J Radiol 52:283-287, 2004

4. Lucas DR: Angiosarcoma, radiation-associated angiosarcoma, and atypical vascular lesion. Arch Pathol Lab Med 133:1804-1809, 2009

5. Young RJ, Brown NJ, Reed MW, et al: Angiosarcoma. Lancet Oncol 11:983-991, 2010

6. Qin ZP, Li KL, Li R, et al: Photodynamic therapy of port wine stains-A report of 238 cases. Photodiagnosis Photodyn Ther 4:53-59, 2007

7. Qiu H, Gu Y, Wang Y, et al: Twenty years of clinical experience with a new modality of vascular-targeted photodynamic therapy for port wine stains. Dermatol Surg 37:1603-1610, 2011

8. Tournas JA, Lai J, Truitt A, et al: Combined benzoporphyrin derivative monoacid ring photodynamic therapy and pulsed dye laser for port wine stain birthmarks. Photodiagnosis Photodyn Ther 6:195-199, 2009

9. Kelly KM, Kimel S, Smith T, et al: Combined photodynamic and photothermal induced injury enhances damage to in vivo model blood vessels. Lasers Surg Med 34:407-413, 2004

10. Jerjes W, Upile T, Hamdoon Z, et al: Interstitial PDT for vascular anomalies. Lasers Surg Med 43:357-365, 2011

11. Tardivo JP, Del Giglio A, Paschoal LH, et al: New photodynamic therapy protocol to treat AIDS-related Kaposi's sarcoma. Photomed Laser Surg 24:528-531, 2006

12. Thong PS, Ong KW, Goh NS, et al: Photodynamic-therapy-activated immune response against distant untreated tumours in recurrent angiosarcoma. Lancet Oncol 8:950-952, 2007

13. Chin WW, Heng PW, Thong PS, et al: Improved formulation of photosensitizer chlorin e6 polyvinylpyrrolidone for fluorescence diagnostic imaging and photodynamic therapy of human cancer. Eur J Pharm Biopharm 69:1083-1093, 2008

14. Martinez MI, Sanchez-Carpintero I, North PE, et al: Infantile hemangioma: Clinical resolution with 5\% imiquimod cream. Arch Dermatol 138:881-884, 2002; discussion 4
15. Itinteang T, Brasch HD, Tan ST, et al: Expression of components of the renin-angiotensin system in proliferating infantile haemangioma may account for the propranolol-induced accelerated involution. J Plast Reconstr Aesthet Surg 64:759-765, 2011

16. Itinteang $\mathrm{T}$, Withers $\mathrm{AH}$, Leadbitter $\mathrm{P}$, et al: Pharmacologic therapies for infantile hemangioma: Is there a rational basis? Plast Reconstr Surg 128:499-507, 2011

17. Ni N, Langer P, Wagner R, et al: Topical timolol for periocular hemangioma: Report of further study. Arch Ophthalmol 129:377379,2011

18. Tremaine AM, Armstrong J, Huang YC, et al: Enhanced port wine stain lightening achieved with combined treatment of selective photothermolysis and imiquimod. J Am Acad Dermatol 66:634-641, 2012

19. Phung TL, Oble DA, Jia W, et al: Can the wound healing response of human skin be modulated after laser treatment and the effects of exposure extended? Implications on the combined use of the pulsed dye laser and a topical angiogenesis inhibitor for treatment of port wine stain birthmarks. Lasers Surg Med 40:1-5, 2008

20. Burrows PE, Mulliken JB, Fishman SJ, et al: Pharmacological treatment of a diffuse arteriovenous malformation of the upper extremity in a child. J Craniofac Surg 20 Suppl 1:597-602, 2009

21. Marler JJ, Fishman SJ, Kilroy SM, et al: Increased expression of urinary matrix metalloproteinases parallels the extent and activity of vascular anomalies. Pediatrics 116:38-45, 2005

22. Rosen T: Limited extent AIDS-related cutaneous Kaposi's sarcoma responsive to imiquimod 5\% cream. Int J Dermatol 45:854-856, 2006

23. Fife K, Howard MR, Gracie F, et al: Activity of thalidomide in AIDSrelated Kaposi's sarcoma and correlation with HHV8 titre. Int J STD AIDS 9:751-755, 1998

24. Little RF, Wyvill KM, Pluda JM, et al: Activity of thalidomide in AIDSrelated Kaposi's sarcoma. J Clin Oncol 18:2593-2602, 2000

25. Uldrick TS, Whitby D: Update on KSHV epidemiology, Kaposi sarcoma pathogenesis, and treatment of Kaposi sarcoma. Cancer Lett 305:150162,2011

26. Koontz BF, Miles EF, Rubio MA, et al: Preoperative radiotherapy and bevacizumab for angiosarcoma of the head and neck: Two case studies. Head Neck 30:262-266, 2008

27. Rosen A, Thimon S, Ternant D, et al: Partial response to bevacizumab of an extensive cutaneous angiosarcoma of the face. Br J Dermatol 163: 225-227, 2010

28. De Yao JT, Sun D, Powell AT, et al: Scalp angiosarcoma remission with bevacizumab and radiotherapy without surgery: A case report and review of the literature. Sarcoma 2011:160369, 2011

29. Hofbauer GF, Marcollo-Pini A, Corsenca A, et al: The mTOR inhibitor rapamycin significantly improves facial angiofibroma lesions in a patient with tuberous sclerosis. Br J Dermatol 159:473-475, 2008

30. Haemel AK, O'Brian AL, Teng JM: Topical rapamycin: A novel approach to facial angiofibromas in tuberous sclerosis. Arch Dermatol 146:715-718, 2010

31. Salido R, Garnacho-Saucedo G, Cuevas-Asencio I, et al: Sustained clinical effectiveness and favorable safety profile of topical sirolimus for tuberous sclerosis-Associated facial angiofibroma. J Eur Acad Dermatol Venereol (in press)

32. Dompmartin A, Blaizot X, Théron J, et al: Radio-opaque ethylcelluloseethanol is a safe and efficient sclerosing agent for venous malformations. Eur Radiol 21:2647-2656, 2011

33. Ihara A, Kurita M, Ozaki M, et al: Subcutaneous injection of normal saline prevents cutaneous complications of ethanol sclerotherapy for superficial vascular lesions: An experimental study. Dermatol Surg 37: 1125-1132, 2011 